\title{
The Influence of Molecular Weight over the Ultrathin Films of Biodegradable Polyion Complexes between Chitosan and Poly $(\gamma$-glutamic acid)
}

\author{
Wanpen TaChaboonyaKiat, Takeshi SerizaWa, Takeshi Endo, ${ }^{*}$ and Mitsuru AKaShI ${ }^{\dagger}$ \\ Department of Applied Chemistry and Chemical Engineering, Faculty of Engineering, \\ Kagoshima University, Korimoto 1-21-40, Kagoshima 890-0065, Japan \\ *Research Laboratory of Resources Utilization, Tokyo Institute of Technology, \\ Nagatsuta-cho, 4259, Midori-ku, Yokohama 226-0026, Japan
}

(Received August 23, 1999)

\begin{abstract}
Alternate adsorption is a well-known method for developing the novel ultrathin films of biodegradable polyion complexes. This study revealed variations in the effect of chitosan and poly ( $\gamma$-glutamic acid) $[\gamma$-PGA] molecular weight on the complex formation process. High molecular weight $\gamma$-PGA showed a high adsorption frequency. In contrast, no molecular weight effect was observed in the case of chitosan. Addition of certain organic solvents in the chitosan and $\gamma$-PGA aqueous solution also influenced ultrathin film polyion complex formation.

KEY WORDS Chitosan / Poly ( $\gamma$-glutamic acid) / Biodegradable Polyion Complexes / Alternate Adsorption / Quartz Crystal Microbalance / Ultrathin Film /
\end{abstract}

Chitin/Chitosan is a naturally regenerating polysaccharide extracted from the exoskelatons of crab and shrimp shells. Chitosan is a deacetylated form of chitin. Chitosan plays an important role in various applications such as water treatment, pharmaceutical and medical applications, biotechnology, food processing, ${ }^{1}$ and membrane formation. ${ }^{2}$ The main driving force in the development of new applications for chitosan lies in the fact that this kind of polysaccharide is not only naturally abundant, but also nontoxic and biodegradable. ${ }^{3}$ Furthermore, chitosan is a good cationic polymer which can be formed biopolyion complexes with its anionic derivatives or other natural anionic polymers.

In our previous study, poly ( $\gamma$-glutamic acid) [ $\gamma$-PGA] was selected as a model of natural anionic polymer. ${ }^{4}$ The purpose of the study was to modify the substrate surface by means of alternate deposition of biodegradable naturally occurring polymers. The quartz crystal microbalance (QCM) method is utilized to quantitate the efficiency of complex formation. Moreover, this method is very useful in the formation of electrostatic ultrathin films. ${ }^{5-14}$ The QCM surface is used as an electronic substrate for microfilm formation. QCM provides a novel method for calculating the amount of polymer adsorption and film formation efficiency on substrates by monitoring decrease in frequency. In this paper, molecular weight of $\gamma$-PGA and chitosan in addition to the type of organic solvents and mixed aqueous-solvent ratio, were varied to examine effects on the efficiency of complex formation.

\section{EXPERIMENTAL}

\section{Materials}

$\gamma$-PGA $\left(M_{w} 600000\right)$ and $\gamma$-PGA $\left(M_{w} 1230000\right)$ was kindly gifted by Meiji Seika Kaisya, Ltd., Japan. Chitosan 1000 , chitosan 500 , chitosan 100 which have weight average molecular weight approximately 1400000, 1200000 , and 650000 respectively were purchased from

\footnotetext{
${ }^{\dagger}$ To whom all correspondence should be addressed.
}

Wako Pure Chemical Industries, Ltd., Japan. The numbers 1000,500 , and 100 mean the average viscosity of chitosan which is 1000,500 , and $100 \mathrm{cP}$, respectively. Sodium hydrogen carbonate were purchased from Wako Pure Chemical Industries, Ltd., Japan. The chemical structure of $\gamma$-PGA and chitosan were shown in Figure 1. Formic acid, acetonitrile and acetone were supplied from Nacalai Tesque, Inc., Japan. All chemicals were used without further purification.

Pure water was purified by a Milllipore Milli-Q system containing a $0.22 \mu \mathrm{m}$ Millipak 40 filter at the outlet.

\section{Quartz Crystal Microbalance}

An AT-cut quartz crystal with a parent frequency of $\mathbf{9}$ $\mathrm{MHz}$ coated with gold electrode was used as a substrate. The adsorption of polymer onto gold surface was calculated as a mass increase $(\Delta m)$ with decreasing in frequency $(\Delta F)$ using Sauerbrey's equation. ${ }^{15}$ The frequency was monitored by an Iwatsu frequency counter (Model SC7201) and data were recorded by personal computer (PC-9801DX).

$$
\text { Sauerbrey's equation: }-\Delta F=\frac{2 F_{0}^{2}}{A \sqrt{\rho_{\mathrm{q}} \mu_{\mathrm{q}}}} \times \Delta m
$$

where $F_{0}$ is the QCM parent frequency of $9 \times 10^{6} \mathrm{~Hz}$. A is the electrode area $\left(0.16 \mathrm{~cm}^{2}\right) . \rho_{\mathrm{q}}$ is the density of the quartz $\left(2.65 \mathrm{~g} \mathrm{~cm}^{-3}\right)$ and $\mu_{q}$ is the shear modulus $(2.95 \times$ $10^{11}$ dyne $\mathrm{cm}^{-2}$ ). This equation is reliable when measured in air.

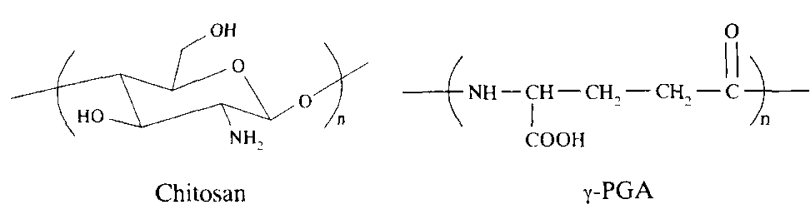

Figure 1. Molecular structure of chitosan and $\gamma$-PGA. 


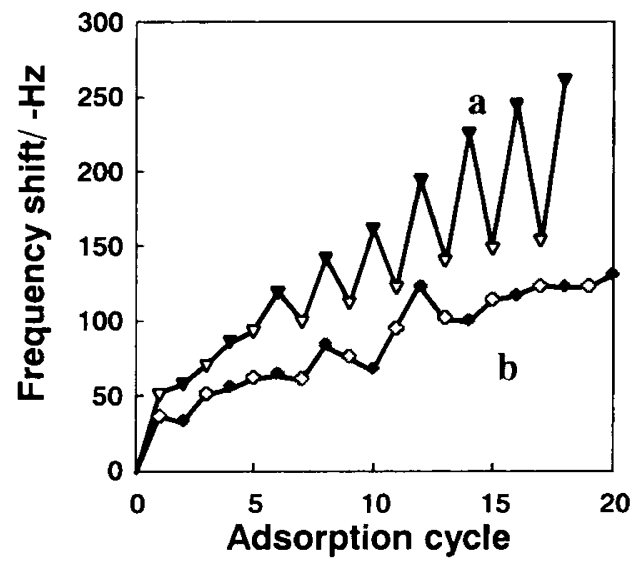

Figure 2. Molecular weight of $\gamma$-PGA influenced on chitosan 500- $\gamma$-PGA polyioncomplex (a) $M_{w} 1230000$ and (b) $M_{w} 600000$. Open and closed symbols show chitosan and $\gamma$-PGA adsorption steps, respectively.

\section{Ultrathin Film Preparation}

The upper electrode wire was protected from the solutions by silicone paint. The QCM surface was treated with piranha solution $\left(\mathrm{H}_{2} \mathrm{SO}_{4}: \mathrm{H}_{2} \mathrm{O}_{2}=3: 1\right)$ for $1 \mathrm{~min}$ two times on both sides, followed by rinsing with pure water and drying with $\mathrm{N}_{2}$ gas. As described in our previous study, ${ }^{4}$ the clean surface of the QCM was immersed in $0.02 \mathrm{M}$ chitosan in $25 \% \mathrm{HCOOH}$ for $5 \mathrm{~min}$, followed by washing with pure water and drying with $\mathrm{N}_{2}$ gas. The frequency decrease indicated the amount of polymer adsorbed. Subsequently, the QCM was immersed in $0.02 \mathrm{M}$ $\gamma$-PGA in $0.02 \mathrm{M} \mathrm{NaHCO}$ followed by washing and drying, using the same procedure. These cycles were alternately repeated to form layer upon layer of polyion complexes. The present study evaluated the effect of the molecular weight of $\gamma$-PGA and chitosan on polyion complex formation.

The organic solvents acetonitrile and acetone were added in each aqueous solution in the specific ratio to facilitate the complex formation.

\section{Atomic Force Microscopy (AFM) Observation}

AFM observation was performed in air by using a Digital Instruments Nanoscope III with a J-type vertical engage piezoelectric scanner and operated in tapping mode.

\section{RESULTS AND DISCUSSION}

The ultrathin biodegradable polyion complexes of the positively charged polysaccharide chitosan alternating with the negatively charged natural compound $\gamma$-PGA were formed on a clean QCM surface. The effect of chitosan and $\gamma$-PGA molecular weight on complex formation was studied. In addition, the ability of certain organic solvents to stabilize ionic interaction was evaluated.

The QCM frequency decreases in proportion to increasing mass on the electrode and provides nanogram sensitivity in mass measurement. The frequency decrease of $1 \mathrm{~Hz}$ corresponds to a mass increase of $0.87 \mathrm{ng}$, in accordance with Sauerbrey's equation. It is our interest to follow the frequency decrease, i.e., polymer mass increase, with increasing numbers of adsorption cycles.

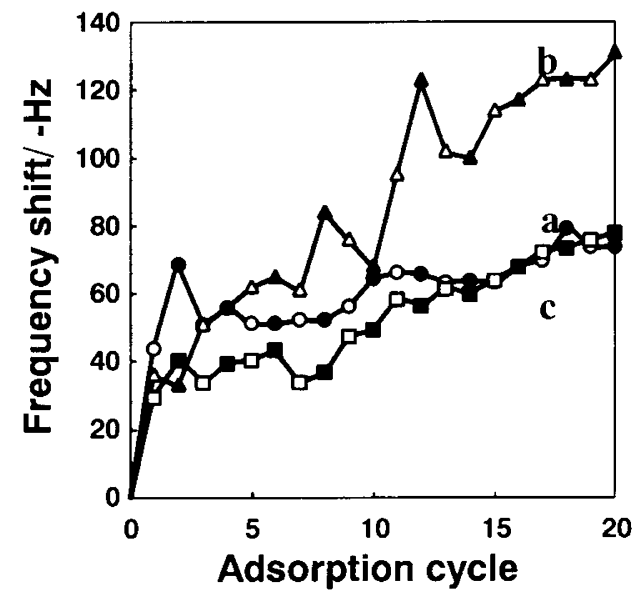

Figure 3. Molecular weight of chitosan influenced on complex formation with $\gamma$-PGA $\left(M_{w} 600000\right)$ (a) chitosan 100 , (b) chitosan 500 , and (c) chitosan 1000.

As shown in Figure 2, high molecular weight $\gamma$-PGA showed higher adsorption. It is well known that polymer adsorption from poor solvent is affected by molecular weight. ${ }^{16}$ The reason of molecular weight effect is unclear. However, one possibility may because of the flexible chain of $\gamma$-PGA. The flexible molecule is a linear chain of atoms connected by chemical bonds. The molecule can change its shape to distribute negative charges by rotation the single bonds in the backbone. The rotation may reduce charge repulsion so that some of these conformations may be favorable. As the molecular weight of $\gamma$-PGA decreased from 1230000 to 600000 caused adsorption frequency decreased from $260 \mathrm{~Hz}$ to $120 \mathrm{~Hz}$. In addition, the present results showed similar adsorption characteristics, when they were compared to data in our previous study ${ }^{4}$ using a stoichiometrically mixed aqueous-acetonitrile solution. On the other hand, Chitosan 500 which has an intermediate molecular weight, gave the highest alternate adsorption with $\gamma$ PGA as shown in Figure 3. The highest molecular weight chitosan (Chitosan 1000) did not show high adsorption frequency. Unlike $\gamma$-PGA, chitosan in aqueous solution showed the limitation of the molecular weight effect, possibly due to not only a large amount of positive charges in a long chitosan 1000 chain, but also the bulky rigid structure of polysaccharide structure caused charge repulsion and stretching out of the molecules. However, chitosan 1000 showed the best polyion complex formation in a mixed aqueous-acetonitrile system (Figure 4). This was attributed to the ability of acetonitrile to interfere with charge repulsion that stabilized the charge effect. Chitosan molecules will be solvated with acetonitrile. The stretch long molecules of chitosan 1000 come close proximity caused by the aggregation of chitosan in poor solvent. This aggregate positively charged chitosan increases an ionic interaction area, resulting in high adsorption onto the substrate.

As shown in Figures 2 and 3, the complexes obtained between chitosan and $\gamma$-PGA in an aqueous solution did not give a well-defined ultrathin film. To develop the ion -ion interaction necessary for ultrathin formation, organic solvents with low solubility were added to chitosan and $\gamma$-PGA aqueous solution in various ratios. 


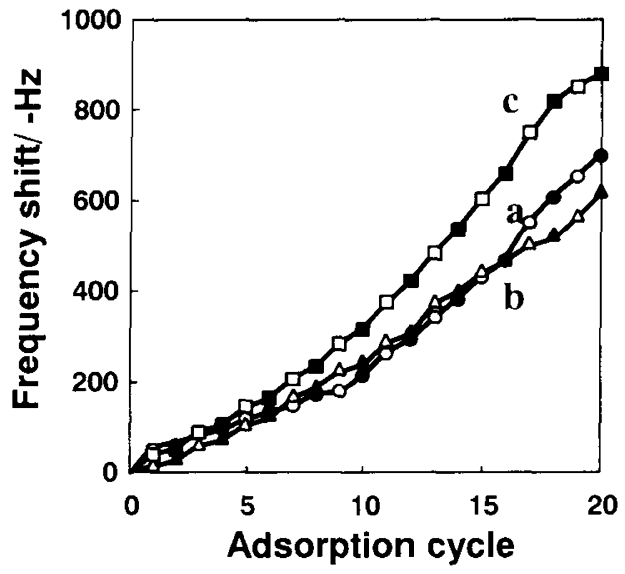

Figure 4. Chitosan- $\gamma$-PGA polyioncomplex with various chitosan molecular weight in mixed aqueous-acetonitrile stoichiometric ratio (a) chitosan 100, (b) chitosan 500, and (c) chitosan 1000.

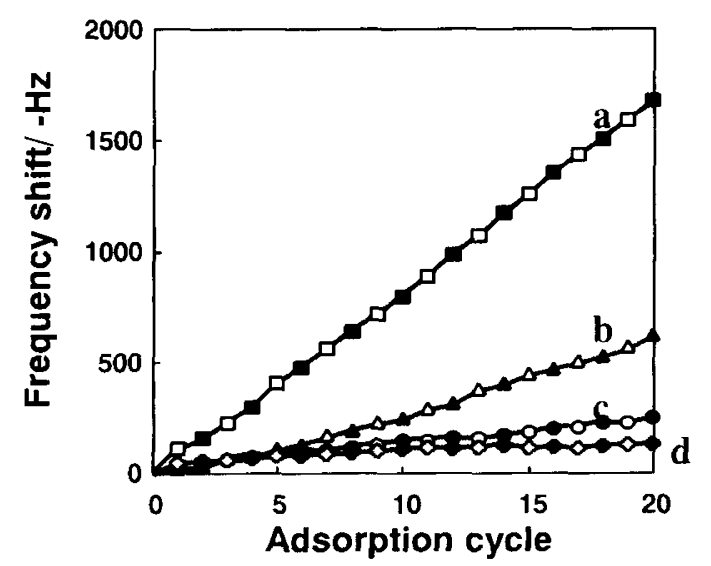

Figure 5. The effect of acetonitrile addition in various ratio on the complex formation (a) aqueous: acetonitrile $2: 3$, (b) $1: 1$, (c) $3: 2$, and (d) $4: 1$.

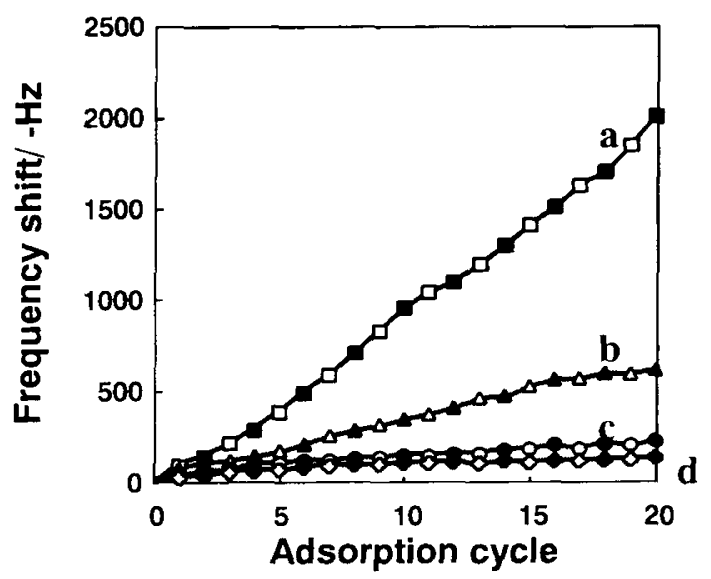

Figure 6. The effect of acetone addition in various ratio on the complex formation (a) aqueous: acetone $2: 3$, (b) $1: 1$, (c) $3: 2$, and (d) $4: 1$.

The aqueous solutions with high solubility readily caused reversible adsorption and desorption reactions referred to as the polymer peel off. The addition of organic solvents into aqueous polymer solutions reduced the solubility of polymer. Organic solvents may enclose
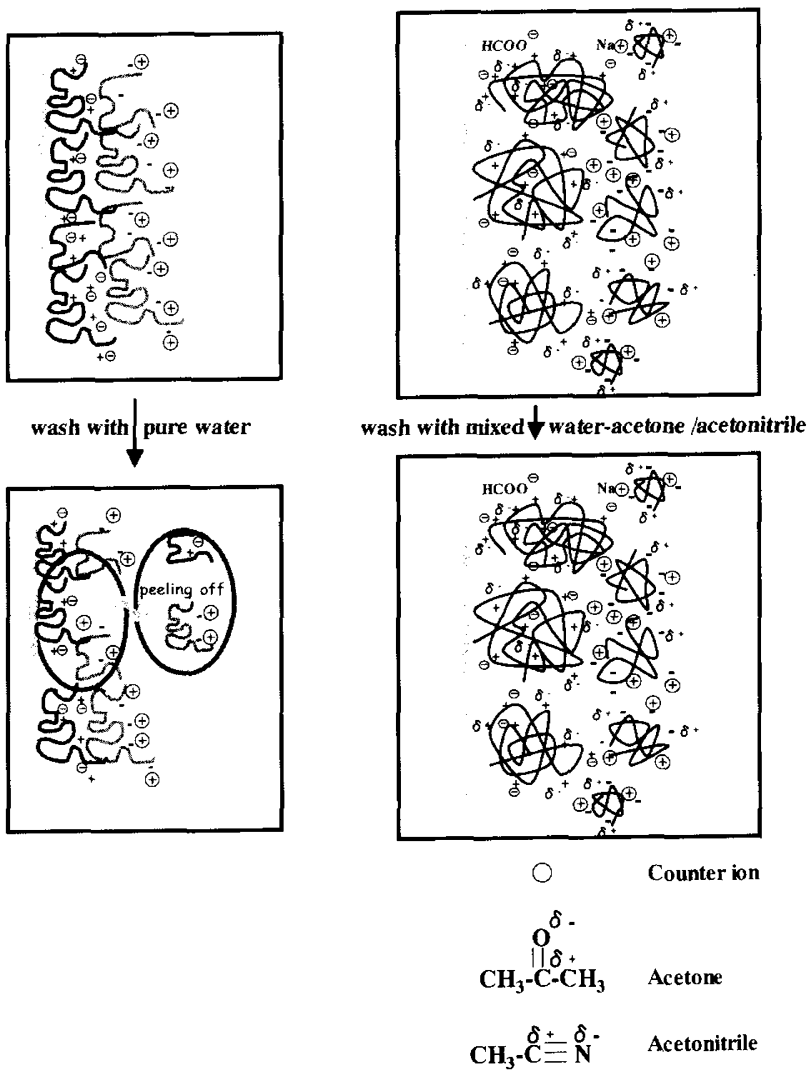

Figure 7. Molecular assembly and molecular orientation model.

cationic or anionic charges and support to stabilize ions to form good polyion complexes, facilitating the deposition process. The addition of increased amounts of low solubility solvents promoted adsorption of greater quantities of the polymer (Figures 5 and 6), indicating that the adsorption was driven by the poor solubility of polymer. These observations suggest that organic solvents with low solubility support formation of good ion-ion interactions between polyion layer. When acetone or acetonitrile was added, each cationic or anionic gathers a cluster of solvents molecules, their positive ends turned toward a negative ion, and their negative ends turned toward a positive ion. The polymer may aggregate into cluster and be adsorbed on QCM surface as well as onto the ionic substrates, effectively increasing the quantity of adsorption. These properties are related to molecular assembly and molecular orientation on the QCM surface as described in Figure 7.

Organic solvents also play an important role in the adsorption process. Organic solvents with high solubility have an increased potential to wash physical adsorption and to cause some parts peel off from the complexes after washing, resulting in low adsorption frequency. A comparison of organic solvents acetonitrile and acetone revealed similar adsorption of polyion complexes in mixed aqueous-acetone as that obtained in mixed aqueous-acetonitrile when small amounts of organic solvent were added. Moreover, better ionic interaction was obtained when organic solvent content exceeded $50 \%$ as shown in Figure 8.

AFM observation was conducted in tapping mode to investigate the surface morphology of the chitosan- $\gamma$ - 


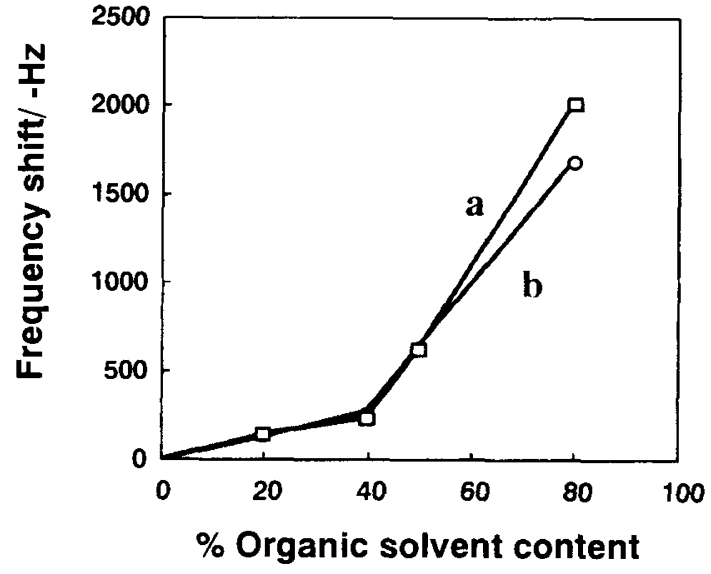

Figure 8. The adsorption frequency after 20 cycles with various organic solvents content (a) mixed aqueous-acetone solution and (b) mixed aqueous-acetonitrile solution.

(a)

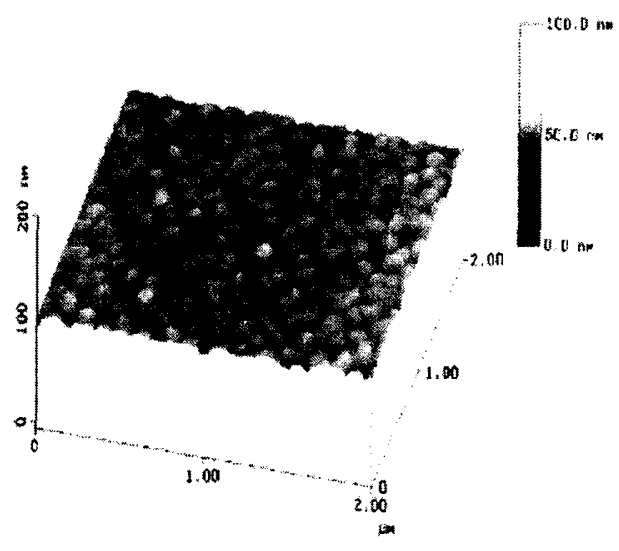

(c)

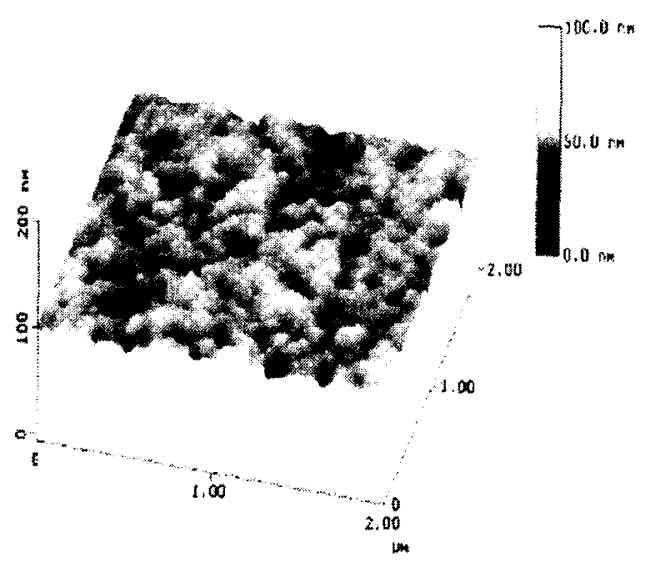

Table I. QCM data and AFM analysis after 20 cycles

\begin{tabular}{lrrrc}
\hline \multicolumn{1}{c}{ System } & $-\Delta F / \mathrm{Hz}$ & $\Delta m / \mathrm{ng}$ & Thickness/nm & Roughness $/ \mathrm{nm}$ \\
\hline Aqueous solution & 131 & 114 & 3 & 0.8 \\
aq.: acetonitrile (2:3) & 1675 & 1457 & 35 & 2.8 \\
aq.: acetone $(2: 3)$ & 2004 & 1743 & 42 & 1.7 \\
\hline
\end{tabular}

Thickness was estimated by assuming the film density 1.3 $\mathrm{g} \mathrm{cm}^{-3}$. (b)

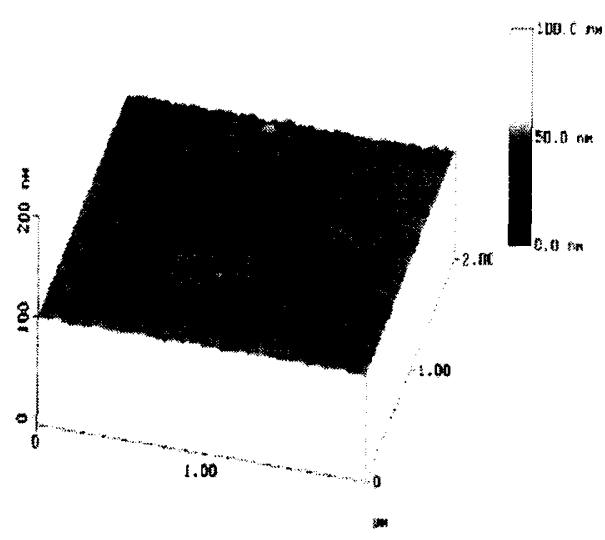

(d)

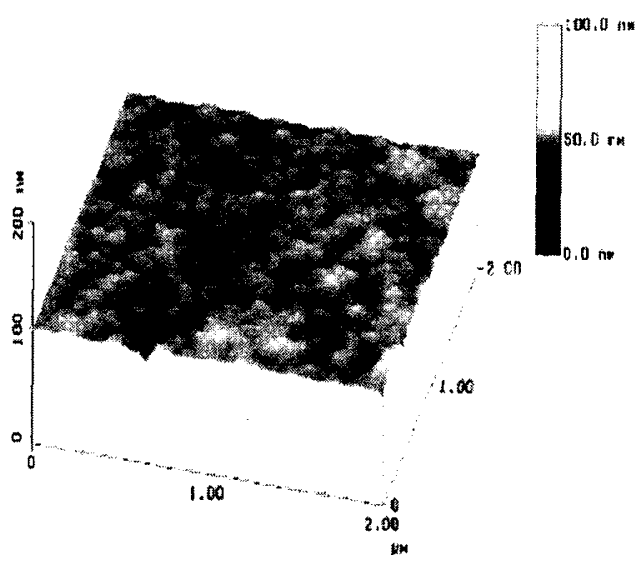

Figure 9. AFM image (a) QCM surface, (b) chitosan- $\gamma$-PGA ultrathin film in aqueous solution, (c) chitosan- $\gamma$-PGA ultrathin film in mixed aqueous-acetonitrile $2: 3$ solution, and (d) chitosan- $\gamma$-PGA ultrathin film in mixed aqueous-acetone $2: 3$ solution.

PGA complexes. The AFM images of alternating chitosan- $\gamma$-PGA adsorption layers under different conditions were observed on gold QCM substrate after 20 cycles as shown in Figure 9. Chitosan- $\gamma$-PGA complexes prepared in aqueous solution showed a small aggregate size (Figure 9b). The polymer which collected into large aggregations of precipitate and which was best adsorbed on the substrate was observed in mixed aqueous acetoni- trile or acetone solution (Figure 9c, 9d). The QCM data and AFM analysis were summarized in Table I. Chitosan- $\gamma$-PGA complexes showed a molecularly smooth surface, ranging in roughness from $0.8-2.8 \mathrm{~nm}$.

\section{CONCLUSION}

Chitosan- $\gamma$-PGA polyion complexes can be develop by 
means of the QCM method and adsorption can be monitored by measuring the decrease in frequency shift. The molecular weight effect of chitosan and $\gamma$-PGA was studied. The molecular weight of $\gamma$-PGA had a direct effect on the frequency shift, with higher molecular weight $\gamma$ PGA causing greater deposition. In the case of chitosan, molecular weight had no effect on the frequency shift. However, the solvent used affected chitosan adsorption. Chitosan-500 in 25\% $\mathrm{HCOOH}$ aqueous solution showed good deposition, but chitosan-1000 in $25 \% \mathrm{HCOOH}$ and acetonitrile in a stoichiometrically mixed solution showed the greatest adsorption. Well-defined ultrathin films could be improved by adding low solubility organic solvents such as acetonitrile or acetone. These organic solvents surrounded the cationic and anionic species to stabilize ionic interaction. High adsorption frequency was related to the molecular assembly onto the substrate.

Acknowledgment. The authors thank the Minister of Education, Science, Sports and Culture, Japan for scholarship to W. Tachaboonyakiat. The authors greatly appreciate to Prof. S. Tokura (Kansai University), not only for his significant help in analysis of the molecular weight of chitosan by HPLC method, but also his advice and suggestions.

\section{REFERENCES}

1. D. Knoor, Food Technol., 85 (1984).

2. N. K. Mathur and C. K. Narand, J. Chem. Ed., 67, 938 (1990),

3. I. M. Laham and T. C. Lee, J. Environ. Polym. Degrad., 3, 31 (1995).

4. T. Serizawa, H. Goto, A. Kishida, T. Endo, and M. Akashi, J. Polym. Sci., Part A: Polym. Chem., 36, 801 (1999).

5. F. Caruso, K. Niikura, D. N. Furlong, and Y. Okahata, Langmuir, 13, 3427 (1997).

6. F. Caruso, K. Niikura, D. N. Furlong, and Y. Okahata, Langmuir, 13, 3422(1997).

7. G. Decher, Science, 227, 1232 (1997).

8. G. Decher, J. D. Hong, and Ber Bunsen-Ges, Phys. Chem., 95, 1430 (1991).

9. A. Delcorte, P. Bertrand, E. Wischerhoff, and A. Laschewsky, Langmuir, 13, 5125 (1997).

10. J. Hodak, R. Etchenique, E. J. Calvo, K. Singhal, and P. N. Bartlett, Langmuir, 13, 2708 (1997).

11. N. A. Kotov, T. Haraszti, L. Turi, G. Zavala, R. E. Geer, I. Dekany, and J. H. Fendler, J. Am. Chem. Soc., 119, 6821 (1997).

12. Y. Lvov, K. Ariga, I. Ichinose, and T. Kunitake, J. Am. Chem. Soc., 117, 6117(1995).

13. Y. Lvov, M. Onda, K. Ariga, and T. Kunitake, J. Biomater. Sci. Polym. Ed, 9, 345 (1998).

14. M. Sano, Y. Lvov, and T. Kunitake, Annu. Rev. Mater. Sci, 26, 153 (1996).

15. G. Z., Sauerbrey, Phys., 115, 206 (1959).

16. G. J. Fleer, M. A. Cohen Stuart, J. M. H. M. Scheutjens, T. Cosgrove, and B. Vincent, "Polymers at Interfaces," 1st ed, Chapman \& Hall, Cambridge, Great Britain, 1993. 